\title{
The problem of objectivity in the Social Sciences: an introduction
}

\author{
[El problema de la objetividad en las Ciencias Sociales: una \\ introducción]
}

\author{
Jose Garcia Martin - Roman Kralik - Miroslav Tvrdon - Zita Jenisova - \\ Lubomir Pekarcik
}

DOI: 10.18355/XL.2022.15.01.13

\begin{abstract}
The aim of this paper is to clarify why objectivity in the social sciences is a problem, what it consists of and to refer to some specific cases of authors who deal with it. Another aim of the presented paper is to address the problem of measurement in the social sciences. Finally, we present our own conclusions and opinions. In any case, it is not intended to be exhaustive or to give a definitive answer, but rather to encourage reflection on analysed problem.
\end{abstract}

Key words: objectivity, values, measurements, validity, reliability

\section{Resumen}

En el presente artículo vamos a aclarar por qué es un problema la objetividad en las ciencias sociales y en qué consiste. Después nos vamos a referir a algunos casos concretos de autores que lo tratan. A continuación, abordaremos el problema de la medida en ciencias sociales. Por último, vamos a plantear nuestra propia posición u opinión a manera de conclusión. En cualquier caso, no se pretende ser exhaustivo ni dar una respuesta definitiva, sino de animar a reflexionar sobre dicho problema de forma introductoria.

Palabras clave: objetividad, valores, medidas, validez, fiabilidad

\section{Introducción}

Antes de abordar el problema planteado, nos gustaría plantear una cuestión propedéutica: cuando hablamos de "objetividad", ¿de qué estamos hablando? Podemos querer significar que se trata de un conocimiento "objetivo", "universal", "válido" y "fiable", independientemente de cualquier circunstancia espacio-temporal o factores socio-culturales. Pero dicha objetividad se apoya en dos presupuestos muy discutibles (Lamo de Espinosa, 2010: 54; Barancova et al., 2018): a) la irrelevancia del sujeto de conocimiento, y b) el carácter racional o inteligible de la realidad, lo cual hace posible su conocimiento. Presupuestos que han sido derruidos por el mismo desarrollo del conocimiento científico y la historia de la ciencia en el s. XX. En términos estrictamente filosóficos, dicho problema equivale, o se corresponde, al problema de la "realidad", en la medida que el conocimiento objetivo es aquel "auténtico" o "verdadero" conocimiento, frente al que no lo es (mera opinión, saber vulgar, conocimiento ordinario o sentido común, Wartofsky, 1978: 90-97); esto es, tiene que ver con el problema de la fundamentación o justificación de nuestros conocimientos. Conceptos, todos ellos, ciertamente cuestionados, pero que necesitan ser tenidos en cuenta o considerados mínimamente, no obstante.

\section{Justificación y delimitación del problema de la objetividad en las CC. SS.}

Lo primero que hay que recordar es que dicho problema no es exclusivo de las ciencias sociales. También está presente en el campo de las ciencias naturales y, en general, en la epistemología o filosofía de la ciencia.

XLinguae, Volume 15 Issue 1, January 2022, ISSN 1337-8384, eISSN 2453-711X 
En el caso de las ciencias formales, matemáticas y lógica, la objetividad cabe entenderla como una cuestión procedimental o de corrección, precisamente por ser "formales"; y también porque modernamente, a partir de Galileo Galilei, se ha entendido que el universo está escrito en lenguaje matemático (o al menos que se puede formular matemáticamente), siendo la realidad esencialmente matemáticas, como en los antiguos pitagóricos $\mathrm{y}$, digamos, en el matematicismo platónico. Se entiende que al existir una simetría perfecta entre matemáticas y naturaleza, o entre la formulación matemática de los fenómenos naturales y la propia realidad natural esencialmente considerada, no debe existir ningún problema sobre un conocimiento objetivo de la naturaleza (algo que fue discutido ya por Gianbattista Vico, 1668-1744, con su Ciencia nueva), o sobre la correspondencia (aunque también depende de cómo se entienda ésta) entre la lógica científica y la lógica natural, o el orden lógicocientífico y el natural. De esta manera, se garantizaba la universalidad y validez del conocimiento científico de la naturaleza. En realidad, la objetividad de las ciencias naturales (en la ciencia moderna clásica) se fundamenta, pues, en el carácter matemático de su conocimiento, por un lado; pero también en la experimentación como el otro pilar fundamental. Y parte del presupuesto de que el "sujeto" y el "objeto" del conocimiento son independientes y están separados, aunque necesariamente involucrados en el proceso del conocimiento como elementos del mismo. La cuestión es que sujeto y objeto de conocimiento están, valga la expresión, hechos el uno para el otro y en perfecta armonía o simetría. Conocimiento sobre la naturaleza o el universo que se "descubre" autoevidente, pero también conocimiento capaz de predecir y controlar la naturaleza en su dimensión práctica. Así pues, se puede concluir que las ciencias naturales son posibles (Akimjakova, 2017).

Sin embargo, el modelo o paradigma del conocimiento tal como lo había entendido la ciencia moderna clásica, se puso en cuestión con la segunda revolución científica acaecida en las dos primeras décadas del siglo XX. La física cuántica, la Teoría de la relatividad de Einstein y el Principio de indeterminación o incertidumbre de Heisenberg, rompieron con dicho paradigma haciendo necesario una nueva manera de entender y explicar el proceso de conocimiento científico. Siguiendo la estela del "giro copernicano" de Kant, la ciencia contemporánea entiende que el "sujeto" de conocimiento (observador, investigador) es elemento activo, y no meramente pasivo, en el proceso de conocimiento. El punto de vista o perspectiva del sujeto de conocimiento con respecto al objeto se torna imprescindible para explicarlo, por lo que la objetividad del conocimiento no se puede entender en términos absolutos, sino relativos (o mejor, "relacionales", como diría Mannheim). Igual que el marco espaciotemporal como referentes absolutos de la física newtoniana es insostenible en la física contemporánea, la objetividad de un conocimiento universal y la propia inteligibilidad o racionalidad de la realidad, tal como la entendía la ciencia clásica moderna, lo son también. En definitiva, cabe preguntarse ahora cómo es posible el conocimiento científico natural (Ambrozy, 2012).

Evidentemente, las ciencias sociales no iban a ser ajenas a toda esta problemática. Aún más si tenemos en cuenta su propio carácter idiosincrático que la distingue de las ciencias naturales: sujeto y objeto de conocimiento no se pueden separar (aunque sí distinguir). Porque en eso se fundamenta básicamente el problema de la objetividad en las ciencias sociales: la estrecha interrelación entre la dimensión objetiva y subjetiva del conocimiento científico social. Los dos paradigmas que nos podemos encontrar al respecto es el "constructivismo" y el "interpretativismo" (Valles, 2007: 59-62), los cuales tienen unos presupuestos ontológicos y unas implicaciones epistemológicas diferentes, presentes, por ejemplo, en el constructivismo social, la hermenéutica o el interaccionismo simbólico. Hasta tal punto que, en cierta manera, uno podría hablar más bien del problema de la "subjetividad" en las ciencias sociales. Problema que ha llevado a cuestionarse si es posible una ciencia social. El cómo y el porqué de ello es lo que tenemos que abordar a continuación. 
Siendo tremendamente concreto, el problema de la objetividad en el área de las ciencias sociales apunta, o se compone, de: a) el problema de los "valores", y b) el problema de la "medida". El primero tiene que ver, sobre todo, aunque no únicamente, con los "juicios de valor" en el conocimiento científico; mientras que el segundo con el problema de la validez y fiabilidad, la generalización y representatividad de los conocimientos científicos sociales, tanto en la investigación cuantitativa como cualitativa. Todo lo cual, insistimos de nuevo, nos lleva a plantearnos si realmente es posible una ciencia social y, si es posible, qué estatuto científico tendría (determinar su objeto y método de estudio).

\section{El problema de los valores en las Ciencias Sociales}

Si queremos formular dicho problema, se podría hacer en los siguientes términos: ¿,es posible conocer (describir y explicar) objetivamente la realidad social sin deformarla? Se entiende que tal "deformación" obedece precisamente a los valores; o más bien, a las valoraciones del observador o investigador social. O con otras palabras más apropiadas o técnicas: ¿es posible evitar el "sesgo" valorativo? A decir verdad, si hacemos depender la objetividad en las ciencias sociales de ello, esto es, aspirar a hacer desaparecer todo y cualquier sesgo en la investigación social, tal tarea es digna de Sísifo e imposible; más bien, a lo que parece que hay que realmente aspirar es a ser conscientes de los sesgos y a controlarlos o reducirlos al máximo, (evitando también las faclacias de cualquier tipo). En ese sentido, nos atreveríamos a afirmar que se podría considerar a todo investigador, en cuanto tal, un sesgo en sí mismo. Queremos decir que, inevitablemente, de una forma u otra, va a estar presente o acompañar la "sombra" del sesgo al investigador. La cuestión es que dicha "sombra" no vele, nuble, oculte o confunda el conocimiento de la realidad social hasta volverla objetivamente irreconocible. A este respecto, cabe decir que la objetividad, más que un problema, es una aspiración; una aspiración legítima y necesaria, pero continuamente inalcanzable o inacabada. Y como los "ídolos" de Bacon, siempre vamos a vernos reflejados en nuestro propio conocimiento de la realidad; siempre va a producirse, a semejanza de un espejo, una cierta refracción o devolución de nuestra propia subjetividad en aquello que pretendemos conocer (Cartwright, Montuschi, 2015).

Pero vayamos al concepto inicial aquí en este punto implicado: ¿qué son los valores? Sin entrar en disquisiciones o averiguaciones profundas, digamos que los valores son cualidades que damos a las cosas, o al contrario, que los poseen las cosas y de las cuales son portadores las cosas. Por tanto, podemos considerar que los valores tienen una fundamentación objetiva o subjetiva, según se consideren de una manera u otra. Por otro lado, los valores pueden ser de distinto tipo o clase: estéticos, éticos, intelectuales, espirituales, materiales o económicos... En cualquier caso, sean cuales sean, las cosas son valiosas porque hay alguien que los valora como tales. La valoración, pues, es un acto subjetivo, se comunique explícitamente o no. Y como tal, está presente implícita o explícitamente en todo lo que hacemos, en nuestro comportamiento (Kondrla, Durkova, 2018). Y más que la cuestión del origen o genealogía de los valores (como quería Nietzsche), el valor de los valores estaría en su significado y función socio-cultural. A este respecto, ante el dilema de si los valores son absolutos o relativos, hay que decir que ni lo uno ni lo otro; esto es, los valores lo son por y para quien pueda apreciarlos, dentro de un contexto o marco históricocultural, y desde una perspectiva teórico-práctica concreta (Akimjakova, Tisovičova, 2017).

Así pues, el científico social no puede sustraerse a la dimensión subjetiva de los valores, de las valoraciones, así como de las creencias o ideas de diversos tipos. La cuestión no es esa; la cuestión es si ello invalida, y si es así cómo y en qué medida, su actividad científica de investigador social. Porque no es lo mismo hablar de "neutralidad ética" en su actividad profesional, que de "avalorismo" o "amoralismo",

XLinguae, Volume 15 Issue 1, January 2022, ISSN 1337-8384, eISSN 2453-711X 
lo cual no es posible. Lo primero debe ser exigible en cualesquiera de los casos, ya que el científico social debe actuar, en cuanto tal, de forma imparcial, haciendo todo lo posible para no dejarse llevar por prejuicios, valoraciones o creencias personales en su investigación. No obstante, eso no significa que no tenga preferencias, valores, ideas o creencias personales, sino que estas no deben "contaminar" su investigación. El avalorismo (carencia absoluta de valores) o el amoralismo (carencia absoluta de moral) no son factibles en cuanto que todo ser humano tiene unos valores y es moral per se (o inmoral; pero no amoral, puesto que nos comportamos o actuamos libremente, no de forma determinada, mecánica o instintiva meramente) (Vymetalova et al., 2021).

Sin embargo, más que en la actividad científica, donde se sustancia el problema de los valores con relación a las ciencias sociales es en su producto o resultado; esto es, en el conocimiento científico social. Como sabemos, dicho conocimiento está compuesto de enunciados, proposiciones o "juicios" sobre "hechos" (sociales). El problema se presenta cuando se mezclan con, o introducen, los "juicios de valor". Si ocurre, el conocimiento científico pierde objetividad y veracidad. ¿Por qué? Pues porque deja de ser consistente, de estar adecuadamente fundamentado en la realidad social estudiada para difuminarse en los sinuosos contornos de nuestra subjetividad. Los juicios de valor se convierten, entonces, en un "caballo de Troya" que aniquila toda garantía de un conocimiento objetivo sobre la realidad social (o natural); o, al menos, contribuye a proyectar la sospecha o suspicacia de que no lo es.

Pero ilustremos brevemente a continuación el problema de la objetividad con dos grandes figuras en el campo de la sociología; si la primera representa (M. Weber) uno de los denominados "padres" fundadores de la sociología, la segunda (H. Martineau) podemos considerarla como su "madre" fundadora. Ambos resaltan la importancia del significado o comprensión de la realidad social. Igualmente, la objetividad para ambos es una cuestión metodológica. M. Weber (1864-1920) se interesó especialmente por la cuestión de la relación de los valores con la ciencia social en su obra La "objetividad" del conocimiento en la ciencia social y en la política social (también trata dicho tema en su ensayo "El sentido de la libertad de valoración en las ciencias sociológicas y económicas"). Según Beltrán (2001: 16; Valco et al. 2021), la respuesta del sociólogo alemán es extensa y compleja, en la cual hay considerar cuatro aspectos diferentes: a) la crítica técnica, b) la separación ser - deber ser; c) la necesaria impregnación de los valores en la tarea investigadora; d) el carácter "instrumental" o metodológico de los valores, ya que su utilización en la investigación social es imprescindible (el aspecto más destacable, problemático y complejo). Para Weber, la ciencia social es una de las ciencias de la cultura. (Wagner, 2019). Como tal, la realidad empírica relevante es la realidad cultural. En ese sentido, su objeto de estudio es, pues, cultural. (Kobylarek et, al. 2020, 2021; Akram et al. 2021). Más concretamente, la realidad social culturalmente significativa. Ahora bien, esto implica tomar en consideración los valores, ya que la relación entre la realidad social objeto de estudio y los valores o ideales sociales es la que la vuelve significativa, y por lo cual también exige su comprensión. Todo lo cual hace que no sea posible una ciencia social libre absolutamente de valores. Sin embargo, esta dimensión subjetiva no anula o pone en cuestión la objetividad de la ciencia social: “(...) el conocimiento de las ciencias culturales, tal como lo entendemos aquí, se halla ligado a una presuposición «subjetiva» en el sentido de que se ocupa de aquellos elementos de la realidad que tienen alguna relación - aunque sea indirecta - con acontecimientos a los que le atribuimos un significado cultural. Pero, a pesar de ello, es un conocimiento estrictamente causal, exactamente en el mismo sentido en que lo es el conocimiento de acontecimientos de la naturaleza individuales y significativos, (...)" (Weber, 2017: 125-126). No obstante, no podemos confundir la causalidad natural o física, de la social. La primera implica determinación; en cambio, la segunda implica comprensión 
de la acción social en base de las intenciones de los actores o agentes sociales involucrados en un hecho o fenómeno social. (Havelka, 2020).

El investigador social, pues, va a trabajar necesariamente con valores o juicios de valor (por ejemplo, abordando su coherencia o ausencia de contradicción interna con respecto a lo que se formula o expresa en ellos). Pero eso no quiere decir que no tenga un juicio crítico respecto a ellos. La cuestión de ser partidario de unos valores o de otros es una cuestión personal del científico social (ídem, p. 77); y, en cualquier caso, la ciencia empírica social no está para decirle a nadie cuáles deben ser sus valores o lo que debe hacer (ídem, p. 78). Así pues, no debe confundirse la neutralidad ética del investigador con su indiferencia moral, la cual es imposible. Es más, "los valores dominantes en el investigador y en su época determinan lo que se convierte en objeto de investigación y hasta dónde se extiende esta investigación en su búsqueda de una explicación causal dentro de las infinitas relaciones de causalidad" (p. 128). En este sentido, la referencia a valores hay que entenderla como una cuestión de selección o instrumental, además de metodológica.

El tema de la validez epistemológica del conocimiento en sociología también fue abordado por la "madre" fundadora de la sociología: Harriet Martineau (1802-1876). Martineau se propuso crear una ciencia de la sociedad basada en la observación empírica que permitiera tomar decisiones políticas y personales guiadas por una comprensión científica de los principios que gobiernan la vida social. Para ello tuvo que desarrollar y proponer unas reglas de la investigación científica social (adelantándose a las famosas reglas del método sociológico propuestas por E. Durkeim), así como su comprensión de la sociedad. El primer aspecto se encuentra en su obra How to observe Morals and Manners; el segundo, en su obra Society in America (ambas de 1838, publicadas tras su viaje a los EE. UU.). Martineau entiende la labor del investigador científico social de la misma manera que la del científico natural: describir y explicar. Pero, en su caso, se trata de observar y explicar la relación entre morals y manners, es decir, entre los valores y normas sociales sobre lo que está o no bien visto, y los modales, costumbres o pautas de comportamiento en una sociedad dada. En ese sentido, su metodología consiste en buscar objetivaciones (manifestaciones particulares de rasgos universales de la vida social, como los enterramientos, suicidios, epitafios) que sean representativas de las costumbres y moral estudiadas (Lengermann y Niebrugge, 2019: 70), a partir de las cuales se puedan establecer generalizaciones.

Sin embargo, la labor del sociólogo para Martineau va más allá de una metodología científica estrictamente natural. Como posteriormente sucederá con M. Weber, Martineau parece defender y proponer más bien un enfoque u orientación "comprensiva" e interpretativa en sociología. Y es así porque hace de la capacidad de "empatizar" un rasgo característico y diferenciador del sociólogo del resto de científicos naturales. Capacidad que, junto con la "crítica" (también presente en Weber) y la "imparcialidad", fundamentan la objetividad del conocimiento sociológico en nuestra autora: "Para Martineau, la validez epistemológica del conocimiento sociológico depende de tres prácticas interconectadas: imparcialidad, crítica y empatía" (idem, p. 68). Ser objetivo significa, precisamente, ser crítico e imparcial. Crítico porque el sociólogo no puede, o no debe, ser un mero observador de la sociedad, sino comprometerse personalmente por su mejora (como lo estuvo la propia autora con la causa por la igualdad de la mujer con respecto al hombre o la abolición de la esclavitud). Imparcial no porque le sea indiferente moralmente los problemas sociales, sino porque debe estudiarlos libre de prejuicios, huyendo de cualquier visión sesgada o interesada, de tal manera que mantenga una "asepsia" en su actividad investigadora empática y crítica, así como una independencia en la aplicación de unos criterios epistemológicos y metodológicos. Escribe Martineau en el párrafo quinto del capítulo I de How to observe Morals and Manners: "Here then is

XLinguae, Volume 15 Issue 1, January 2022, ISSN 1337-8384, eISSN 2453-711X 
the wise traveller's aim, -- to be kept in view to the exclusion of prejudice, both philosophical and national. He must not allow himself to be perplexed or disgusted by seeing the great ends of human association pursued by means which he could never have devised, and to the practice of which he could not reconcile himself." Y casi al principio del capítulo III afirma. "An observer, to be perfectly accurate, should be himself perfect. Every prejudice, every moral perversion, dims or distorts whatever the eye looks upon".

\section{El problema de la medida en las ciencias sociales}

Llegado a este punto, puede entenderse que preocupe de forma especial la objetividad más en la investigación sociológica de tipo cuantitativo, que en la cualitativa. En este último caso, las diversas técnicas empleadas (entrevistas abiertas, grupos de discusión, hechos de vida, etc.), buscan sobre todo la "comprensión" por encima de la "explicación". No obstante, desde el punto de vista cuantitativo, es posible traspasar, verter o convertir aquello que se desea comprender e interpretar a datos cuantitativos. Incluso actitudes y opiniones; para ello bastaría el uso de alguna escala de medida, como la escala sumativa tipo Likert o las escalas de magnitudes (por ejemplo, la escala de Prestigio Profesional para España PRESCA 2 de Carabaña Morales y Gómez Bueno, 1996). También es posible aplicar la cuantificación mediante el recurso a los índices e indicadores (descriptivos y analíticos), los cuales nos permiten registrar los datos a partir de las dimensiones características o definitorias del fenómeno social objeto de estudio o investigación. Todos estos instrumentos de medición nos permiten asignar magnitudes o valores numéricos preestablecidos a la dimensión social cualitativa objeto de investigación. La cuestión, en definitiva, es si es posible medir en ciencias sociales, puesto que "desde una perspectiva crítica se ha puesto en entredicho la posibilidad de medición objetiva" (Callejo Gallego, 2010: 217).

Hay que tener en cuenta que en la investigación social se trabaja con datos; datos que se producen aplicando alguna de las técnicas de investigación a partir de la información convenientemente recogida sobre el hecho o fenómeno social objeto de estudio. En ese sentido, debemos de ser conscientes de que entre el hecho social observado y las conclusiones obtenidas en su estudio o conocimiento, media una serie de pasos o factores intervinientes; más concretamente, todo lo que tiene que ver con el proceso de investigación social (Quivy, R. y Van Campenhoudt, L. 1992: 16-23; Santana Leitner, 2013: 15-22) que pueden afectar en mayor o menor medida a su objetividad. Así pues, parece que siempre es posible medir y por tanto cuantificar. La cuestión estaría en el "cómo", esto es, en el procedimiento, técnicas, instrumentos y criterios aplicados o utilizados.

Volviendo al principio, al problema de la medida en las CC. SS., éste presenta una doble cara o vertiente. Por un lado, el problema de la validez de las medidas; por el otro, el problema de la fiabilidad de las medidas. Empezando por esta última, hay que decir que la misma se refiere a la "calidad", "precisión" o "exactitud" de la medición. La cual, a su vez, tiene relación y depende de los instrumentos y técnicas empleadas en la misma que eviten los posibles sesgos o errores (aleatorios o sistemáticos) en la medición y, por tanto, en los resultados obtenidos en nuestra investigación. Ahora bien, la fiabilidad exige determinar unos criterios con respecto a los instrumentos de medida, así como calcularla o estimarla mediante unos coeficientes que sirvan para comprobarla. La estabilidad de una medida se comprueba tras su aplicación en repetidos casos (el más conocido método es el de "test-retest"). La equivalencia consistiría en comprobar resultados equivalentes después de aplicar paralelamente a un mismo caso el mismo instrumento de medición. Por su parte, la consistencia implica aplicar un mismo instrumento de medida a dos subconjuntos de una muestra (método de las dos mitades) con respecto a una característica o variable, de tal manera que el coeficiente de correlación determinaría el grado de fiabilidad (Palacios Gómez, 
2014: 39-41). Obviamente, estaríamos hablando de la consistencia interna de la medición.

En cuanto a la validez, tiene que ver con la cuestión de si realmente medimos con un instrumento lo que decimos o pretendemos medir. No es algo que tenga directamente nada que ver con dicho instrumento, sino con la interpretación o inferencia que se haga a partir de los datos producidos en nuestra investigación. De cualquier manera, podemos diferenciar distintos tipos de validez (aunque no existe una unanimidad en una misma clasificación): validez interna y externa, validez de constructo y validez estadística, siendo la primera la distinción principal en la que se basa las otras dos clases de validez. En el caso de la validez interna, se pretende garantizar la objetividad evitando o eliminando cualquier posibilidad de interpretaciones alternativas a la nuestra. Especialmente importante es la validez de constructo, ya que tiene que ver con la coherencia entre nuestro marco conceptual en el que nos basamos en la investigación y los hechos observados. En cambio, la objetividad de la validez externa se refiere a la posibilidad de que los resultados o conclusiones a los que hemos llegado en nuestra investigación puedan generalizarse más allá del marco temporal dado. Y en la medida en que se haga uso de la estadística, habría que hablar de validez estadística; concretamente de estadística inferencial, la cual consiste en proyectar las conclusiones obtenidas en una muestra a la población en su conjunto que se pretende estudiar, apoyándose en la Teoría de Muestras, en el Cálculo de Probabilidades y en la Estadística inferencial (Espejo et al., 2009: 1-5, UCA). En cualquier caso, la validez estadística depende desde la fortaleza del test que se emplee, hasta la heterogeneidad aleatoria de las unidades de análisis o sujetos que se investiguen.

En definitiva, tanto la validez como la fiabilidad son dos aspectos metodológicos que intentan dar respuesta al problema de la objetividad en su vertiente práctica. Aunque no de una manera definitiva o con una garantía absoluta. Algo, por otro lado, que no nos debe sorprender si tenemos en cuenta que todo conocimiento humano es, por ello mismo, limitado e imperfecto. Lo cual no es óbice para que no aspire a ser un conocimiento cada vez más perfecto.

\section{Conclusiones}

Respecto al problema de los valores, sea cual sea su estatuto ontológico, parece evidente que éstos necesariamente van a estar presentes en cualquier actividad humana $\mathrm{y}$, por tanto, también en la científica, sea natural o social. Su dimensión humana y subjetiva no tiene por qué invalidar necesariamente la debida imparcialidad del científico social. Sobre todo, cuando de lo que se trata es de poder hacer una valoración crítica sobre la realidad social en estudio. Sin embargo, resulta imprescindible que tal estudio se fundamente en algún tipo de metodología científica, ya sea cuantitativa o cualitativa; o mejor aún, y en la medida de lo posible, en una combinación de ambas. Por otro lado, y en cualquier caso, deberíamos tomar en consideración el origen social de los mismos valores, ideas o creencias (en definitiva, del conocimiento y la cultura en una sociedad dada) (Akhram et al., 2021; Bakhov et al., 2021; Lojan, 2019; Hubkova 2019; Pekarcik 2020).

En ese sentido, y huyendo tanto del dogmatismo como del relativismo, nos parece interesante la propuesta de la sociología del conocimiento de K. Mannheim (18931947) en su obra Ideología y utopía (1973: 285-289), según la cual debemos relacionar y particularizar el estudio de nuestras ideas con los diversos intereses de los grupos o clases sociales en una constante lucha ideológica por imponerlas a los demás; por eso, los valores, como el conocimiento sociológico del propio conocimiento, y como el conocimiento de las partículas subatómicas, o de los hechos espacio-temporalmente considerados, es una cuestión dependiente de la "perspectiva" y situación del observador (Akimjak 2015; Bielak et al., 2016, Hammersley, 2021).

XLinguae, Volume 15 Issue 1, January 2022, ISSN 1337-8384, eISSN 2453-711X 
Ahora bien, resulta discutible y problemático concebir lo que es un "hecho" (natural, pero sobre todo social). Y lo es porque hablar de "hecho" (natural o social) presupone una cierta concepción de la realidad (natural o social). Parece evidente que hay que desechar la posibilidad ingenua de admitir la existencia de hechos "puros". En la misma medida que también hay que descartar la existencia de un sujeto de conocimiento puro. Sujeto y objeto de conocimiento están estrechamente interrelacionados entre sí e interdependientes. Igual que conocimiento y realidad. De todas maneras, los hechos se constituyen como el objeto de estudio de toda ciencia. El problema está, en este punto, en si podemos hablar en el mismo sentido con respecto a los hechos naturales y sociales. Aquí ya depende de la orientación metodológica: se pueden considerar los hechos naturales y sociales idénticos (Durkheim y, en general, la orientación positivista o naturalista), o bien distintos (Dilthey y, en general, el historicismo y la hermenéutica). O dicho con otras palabras: cabe defender o bien un "monismo" metodológico, o bien un "dualismo", puesto que los hechos sociales no son asimilables a los naturales (no obstante, en cuanto a la metodología en la investigación sociológica, la tendencia actualmente es apelar a la pluralidad metodológica o multimodal, siempre que sea posible). En el primer caso, parece viable poderse garantizar la objetividad y la cientificidad de las ciencias sociales; no tanto en el segundo, lo que ha implicado cuestionarse el estatuto científico de las ciencias sociales. (Datta \& Hanemaayer, 2021). Sea como sea, la relación entre teoría y prácticas sociológicas, entre teoría y métodos sociológicos, ha sido un tema y una fuente constante de problemas (algo que trata muy adecuadamente el texto de Hughes y Sharrock, 2007).

Finalmente, la validez de los datos y la fiabilidad de los instrumentos de medida, es un problema relacionado con las técnicas de investigación cuantitativa. En cuanto tal, tiene que ver con todo el proceso de recogida de información, procesamiento, cuantificación, operacionalización y generalización, usando la estadística y el cálculo de probabilidades. A este respecto, aparte de evitar en la medida de lo posible cualquier tipo de sesgo y falacias, sería conveniente la continua revisión, no solo por parte del propio investigador, sino también por parte de los demás expertos o especialistas; de tal manera que fuera el "carácter público" la que diera plausibilidad de veracidad a dicho estudio o investigación (Giddens, 1993: 55). Visto lo cual, parece que no queda más remedio que admitir cierto carácter de provisionalidad a la pretendida objetividad del conocimiento científico. Algo, por otro lado, consustancial tanto a nuestras limitadas capacidades cognitivas como a nuestros métodos, al procedimiento de investigación e instrumentos científicos.

\section{Acknowledgment}

The work was supported by the Cultural and Educational Grant Agency (KEGA) of the Ministry of Education, Science, Research and Sports of the Slovak Republic based on project "Implementation of e-learning in the chemistry education for applied ecology and environmental science", number 029UKF-4/2020

\section{Bibliographic references}

Akimjak, A. (2015). Sociálna filozofia. Levoča: MTM. ISBN 978 80-89736-38-6.

Akimjakova, B. (2017). Environmentálna výchova prostredníctvom zážitkového učenia. Pedagogické diskusie 3(4), pp. 59-72. ISSN 1339-9217.

Akimjakova, B. \& Tisovicova, A. (2017). A man and values in historical and educational reflection. Revue Internationale des Sciences humaines et narurelles 7(3), pp. 91-100. ISSN 2235-2007.

Akram, H., Yingxiu, Y., Al-Adwan, A. S., \& Alkhalifah, A. (2021). Technology Integration in Higher Education During COVID-19: An Assessment of Online Teaching Competencies Through Technological Pedagogical Content Knowledge Model. Frontiers in Psychology 12, 73652. 
Ambrozy, M. (2012). Some aspects of utilization of anthropological principle arguments in theologia naturalis in the light of analytical philosophy of religion. New Developments in Anthropology Research, pp. 83-90.

Bakhov, I., Opolska, N., Bogus, M., Anishchenko, V., \& Biryukova, Y. (2021). Emergency distance education in the conditions of COVID-19 pandemic: Experience of Ukrainian universities. Education Sciences 11(7), p. 364.

Barancova, M., Birova, J., \& Kralova, Z. (2018). Vplyv sociálno-demografických faktorov na výskyt kontaktových javov v reči Slovákov žijúcich v Spanielsku. Jazykovedny casopis 69(2), pp. 175-186.

Beltrán, M. (2001). Ciencia y sociología. Madrid: CIS.

Bielak, J., Majda, P., Jurko, J., \& Budayova, Z. (2016). The Theology of Social Work in the Chosen Volumes of Slovak authors. Dublin: International Scientific Board of Catholic. ISBN 978-0-9957986-0-1.

Cartwright, N. \& Montuschi, E. (ed.) (2015). Philosophy of Social Science: A New Introduction. Oxford University Press. ISBN 978-0199645107.

Callejo Gallego, J. (ed.). (2010). Introducción a las técnicas de investigación social. Madrid: Editorial universitaria Ramón Areces.

Carabaña Morales, J. \& Gomez Bueno, C. (1996). Escalas de prestigio profesional. Cuaderno Metodológico no 19. Madrid. CIS.

Datta, R.P. \& Hanemaayer, A. (2021). Nominalist visualities and classical social theory: An examination of Durkheim and Weber. Journal for the Theory of Social Behaviour 51(4), pp. 565-586. https://doi.org/10.1111/jtsb.12281

Dolzhikova, A., Kurilenko, V., Biryukova, Y., Baryshnikova, E., Shcherbakova, O., \& Glazova, O. (2021). Why did they keep silent? Some peculiarities of intercultural academic communication. Intercultural Education 32(1), pp. 83-99.

Espejo et al. (2009). Inferencia estadística. Cádiz: Editorial UCA.

Giddens, A. (1993). Sociología. Madrid: Alianza Editorial.

Hammersley, M. (2021). Karl Mannheim's Ideology and Utopia and the public role of sociology. Journal of Classical Sociology. https://doi.org/10.1177/1468795X20986382

Havelka, O. (2020). Teologicko-etický princip personality a jeho soudobé myšlenkové směry. Verba Theologica 19(2), pp. 98-109. ISSN 1336-1635.

Hubkova, S. (2019). Social cultural context with ict development. Revue Internationale des Sciences humaines et naturelles 10(1), pp. 55-63. ISSN 2235-2007.

Hrabakova, E. V., Kondrla, P., Vlasova, V. K., Dmitrichenkova, S. V., \& Pashanova, O. V. (2020). Human as the protector of creation. XLinguae 13(3), pp. 13-21. Doi: 10.18355/XL.2020.13.03.02

Hughes, J A. \& Sharrock, W. W. (2007). Theory and Methods in Sociology. An Introduction to Sociological Thinking and Practice. London, New York: Palmgrave MacMillan. ISBN 978-0-333-77285-0.

Kobylarek, A., \& Błaszczynski, K. (2020). East european u3as facing the problem of seniors' needs. Revista Lusofona de Educacao 49(49), pp. 27-40.

Kobylarek, A., Błaszczynski, K., \& Alam, S. (2021). Science in retrotopia. XLinguae 14(3), pp. 29-36. Doi: 10.18355/XL.2021.14.03.03

Kondrla, P., \& Durkova, E. (2018). Interpersonal relations in ethics of science and technologies. Communications - Scientific Letters of the University of Zilina 20(1PartA), pp. 45-50.

Lamo de Espinosa, E., Gonzales Garcia, J. M., \& Torres Albero, C. (2010). La sociología del conocimiento y de la ciencia. Madrid: Alianza Editorial.

Lelgermann, P. M., \& Niebrugge, G. (2019). Fundadoras de la sociología y la teoría social 1830-1930. Madrid: CIS.

XLinguae, Volume 15 Issue 1, January 2022, ISSN 1337-8384, eISSN 2453-711X 
Lojan, R. (2019). Material and non-material poverty of seniors. Revue Internationale des Sciences humaines et naturelles 10(4), pp. 19-25. ISSN 2235-2007.

Mannheim, K. (1973). Ideología y utopia. Madrid: Aguilar.

Martineau, H. (2010). [1838]. How to observe Morals and Manners. The Project Gutenberg EBook of How to Observe, by Harriet Martineau. [EBook \#33944]. Produced by Julia Miller and the Online Distributed Proofreading Team at http://www.pgdp.net.

Palacios Gomez J. L. (2014). Técnicas cuantitativas de investigación social. Madrid: Editorial Universitas.

Pekarcik, L. (2020). Family as the first social educational environment child and school as a second socialization environment. Revue Internationale des Sciences humaines et naturelles 11(3), pp. 155-167. ISSN 2235-2007.

Quivy, R, \& Van Campendoudt, L. (1992). Manual de investigación en ciencias sociales. México: Limusa.

Santana Leitner, A. (2013). Fundamentos para la investigación social. Madrid: Alianza editorial.

Valco, M., Sturak, P., Pavlikova, M., \& Pala, G. (2021). John Paul II's legacy as a resource for fighting totalitarianism: Slovak experience. Philosophia (Philippines) 21, pp. 429-452.

Valles, M. S. (2007). Técnicas cualitativas de investigación social. Madrid: Editorial Síntesis.

Wagner, P. (2019). Max Weber and 21st-Century Modern. Sociologiceskoe Obozrenie 18(4). DOI 10.17323/1728-192x-2019-4-212-230.

Wartofsky, M. W. (1978). Introducción a la filosofía de la ciencia. 1978. Madrid: Alianza Editorial.

Weber, M. (2017). La "objetividad" del conocimiento en la ciencia social y en la política social. Madrid: Alianza Editorial.

Weber, M. (1993). Sobre la teoría de las ciencias sociales. Barcelona: Editorial Planeta-De Agostini. 230.

Words: 5854

Characters: 38436 (21,35 standard pages)

Prof. Dr. Jose Garcia Martin

Department of Sociology

Faculty of Political Sciences and Sociology

University of Granada

C/Rector López Argüeta, S/N

18007 Granada

Spain

jgarciamartin@ugr.es

Prof. ThDr. PaedDr. Roman Kralik, ThD. (Correspondence author)

Russian language department

RUDN University, Miklukho-Maklaya street, 6

117198 Moscow

Russia Federation

roman.kralik73@gmail.com

Faculty of Theology

Spisske Podhradie - Dpt. Social Work

The Catholic University in Ružomberok

03401 Ružomberok

Slovakia 
Assoc. Prof. PaedDr. Miroslav Tvrdon, PhD.

Constantine the Philosopher University in Nitra Tr. A. Hlinku 1

94901 Nitra

Slovakia

mtvrdon@ukf.sk

Assoc. Prof. Zita Jenisová, PhD.

Constantine the Philosopher University in Nitra

Tr. A. Hlinku 1

94901 Nitra

Slovakia

zjenisova@ukf.sk

Prof. ThDr. Lubomír Pekarcik, PhD.

Faculty of Theology

The Catholic University in Ruzomberok

03401 Ruzomberok

Slovakia

lubomir.pekarcik@ku.sk 